\title{
Suicidality in children and adolescents: lessons to be learned from the COVID-19 crisis
}

\author{
Pieter J. Hoekstra ${ }^{1}$ \\ Published online: 2 June 2020 \\ ○) Springer-Verlag GmbH Germany, part of Springer Nature 2020
}

Suicide is the second most prevalent cause of death worldwide overall and the third leading cause of death in 15-19-year-olds. The most frequent suicide method worldwide is hanging, followed by poisoning by pesticides for females and firearms for males [1]. For every suicide, there are many more people who attempt suicide every year. A prior suicide attempt is the single most important risk factor for suicide in the general population [2]. Mortality and sociodemographic factors data from the Swiss National Cohort (involving the whole Swiss resident population) indicated a clearly increasing suicide rate with increasing age, from none per 100,000 at age 10 years to 14.8 per 100,000 at 18 years in boys, and 5.4 per 100,000 in girls [3]. Other risk factors, apart from male sex, were living in a single parent household, being an only or middle-born child, and living in rural regions. Prevalence of suicide in children and adolescents was remarkably stable over the years 1991-2003, in contrast to a decreasing trend in adults [4].

In this month's issue of European Child and Adolescent Psychiatry, figures from a general population cohort of 1035 junior high-school students (mean age of 13.2 years) from Taiwan showed that suicidal ideation was observed in $20.3 \%$, suicide plans in $5.7 \%$, and actual suicide attempts in $4.7 \%$ during the course of one year [5]. Students with suicidal behaviors were more likely to be females and to be living in highly urbanized areas. Perceived stress as measured by the ten-item perceived stress scale appeared a major predictor of suicidality. Although a measure of resilience did indicate protective effects, the effect sizes of protective factors were smaller than those of risk factors. In a previous study, conducted in a large population cohort of seventh and tenth grade students from Korea, family conflict, being exposed to

Pieter J. Hoekstra

p.hoekstra@accare.nl

1 Department of Child and Adolescent Psychiatry, University Medical Center Groningen, University of Groningen, Groningen, The Netherlands violence, and being victim of bullying were associated with an increased risk of suicidal ideation [6].

A systematic review into psychosocial risk factors for suicidality in children and adolescents, also in the current issue of European Child and Adolescent Psychiatry [7], substantiate the role of stressful life events (in interaction with vulnerability factors), most notably family conflicts, academic stressors (including bullying or exam stress), trauma, and other adverse life events (peer conflict, legal problems, physical abuse, worries about sexual orientation, romantic breakups, exposure to suicide/suicide attempts, and physical and/or sexual violence). Temperament and character are also an important factor; of note, $50 \%$ of adolescents have only started thinking about self-harm less than an hour before the act itself.

Apart from psychosocial stressors, also the presence of mental disorders clearly increases the risk of suicide attempts. Based on data from a 10-year prospective study of 3021 community subjects (14-24 years of age), almost all mental disorders were associated with an elevated risk of suicide attempts [8]; the more mental disorders a subject had, the higher the risk of a suicide attempt. The highest risks of suicide attempts were associated with posttraumatic stress disorder, dysthymia, panic disorder, generalized anxiety disorder, obsessive-compulsive disorder, agoraphobia, and nicotine dependence. As the authors rightly noticed, most of these disorders are well treatable and may thus form promising early intervention targets to decrease suicidality.

The recent measures imposed on families in the current COVID-19 pandemic is unprecedented. An important question is if and how they affect suicide rates in children and adolescents. A higher risk of being exposed to physical and/or sexual violence at home and the economic damage caused by the crisis may well lead to increased suicide rates. There is, however, still a lack of empirical data on the consequences of the COVID-19 crisis on children with mental disorders. An early study in China showed that children's ADHD behaviors worsened related to lowered mood of the 
children and their parents [9]. Another study found almost doubled rates of anxiety and depression in adults from the general population who were affected by quarantine [10]. Lower household income, lower education level, being more worried about being infected, having no psychological support, and lower self-perceived health were significantly associated with higher levels of anxiety and depression.

On the other hand, one may argue that school closures have led to less academic and social pressure, and less peer conflict and bullying. This may have a substantial positive impact on the wellbeing of some vulnerable children and perhaps lead to lessened suicidality rates. It will be of utmost importance to learn lessons how schools may use the benefits of online teaching to decrease psychosocial risk factors associated with suicidality and to improve the mental health for large groups of children. It is too early to tell how suicide rates have developed during lockdown measures, but some countries have reported remarkable decreases (Japan [11], The Netherlands [12], North Rhine-Westphalia in Germany [13], and New Zealand [14]).

The impact of the COVD-19 crisis on child and adolescent practice has also been substantial, with many outpatient assessments and treatments now delivered through videoconferencing. For some families this may lower the barrier of seeking treatment, especially for those with suicidality, depression, and anxiety [15]. Telepsychiatry is also well suited for providing pharmacotherapy in accordance with evidence-based treatment protocols for children with attention-deficit hyperactivity disorder [16]. It is important that there will be an increase in well-powered randomized controlled trials into the effects of telepsychiatry in comparison to conventional modes of treatment delivery. Better use of online treatment delivery could have a lasting positive effect on reaching vulnerable families and on delivering more effective services.

\section{References}

1. Kolves K, de Leo D (2017) Suicide methods in children and adolescents. Eur Child Adolesc Psychiatry 26(2):155-164

2. World Health Organization (2018) Fact sheet of suicide. https:// www.who.int/mediacentre/factsheets/fs398/en/. Accessed 28 May 2020
3. Steck N, Egger M, Schimmelmann BG, Kupferschmid S, Swiss National C (2018) Suicide in adolescents: findings from the Swiss National cohort. Eur Child Adolesc Psychiatry 27:47-56

4. Hepp U, Ring M, Frei A, Rössler W, Schnyder U, AjdacicGross V (2010) Suicide trends diverge by method: Swiss suicide rates 1969-2005. Eur Psychiatry 25(3):129-135

5. Chen YL, Kuo PH (2020) Effects of perceived stress and resilience on suicidal behaviors in early adolescents. Eur Child Adolesc Psychiatry (this issue)

6. Lee D, Jung S, Park S, Hong HJ (2018) The impact of psychological problems and adverse life events on suicidal ideation among adolescents using nationwide data of a school-based mental health screening test in Korea. Eur Child Adolesc Psychiatry 27:1361-1372

7. Carballo JJ, Llorente C, Kehrmann L et al (2020) Psychosocial risk factors for suicidality in children and adolescents. Eur Child Adolesc Psychiatry (this issue)

8. Miche M, Hofer PD, Voss C, Meyer AH, Gloster AT, BeesdoBaum K, Lieb R (2018) Mental disorders and the risk for the subsequent first suicide attempt: results of a community study on adolescents and young adults. Eur Child Adolesc Psychiatry 27:839-848

9. Zhang J, Shuai L, Yu H, Wang Z, Qiu M, Lu L, Cao X, Xia W, Wang Y, Chen R, Zhang J et al (2020) Acute stress, behavioural symptoms and mood states among school-age children with attention-deficit/hyperactive disorder during the COVID-19 outbreak. Asian J Psychiatry 51:102077

10. Lei L, Huang X, Zhang S, Yang J, Yang L, Xu M, Lei L et al (2020) Comparison of prevalence and associated factors of anxiety and depression among people affected by versus people unaffected by quarantine during the COVID-19 epidemic in southwestern China. Med Sci Monit 26:e924609

11. https://www.sbs.com.au/news/japan-s-suicide-rate-plummetsduring-coronavirus-lockdown-while-other-countries-report-incre ase-in-distress-calls. Accessed 28 May 2020

12. https://nos.nl/artikel/2334626-tot-20-procent-minder-zelfdoding en-in-coronatijd.html. Accessed 28 May 2020

13. https://www.augsburger-allgemeine.de/bayern/So-hat-sich-dieSuizidrate-in-Deutschland-waehrend-der-Corona-Krise-entwi ckelt-id57332446.html. Accessed 28 May 2020

14. https://www.nzherald.co.nz/nz/news/article.cfm?c_id=1\&objec tid=12333030. Accessed 28 May 2020

15. Fairchild RM, Ferng-Kuo SF, Rahmouni H, Hardesty D, Fairchild RM et al (2020) Telehealth increases access to care for children dealing with suicidality depression, and, anxiety in rural emergency departments. Telemed J E Health. https://doi.org/10.1089/ tmj.2019.0253

16. Rockhill CM, Tse YJ, Fesinmeyer MD, Garcia J, Myers K, Rockhill CM et al (2016) Telepsychiatrists' medication treatment strategies in the children's attention-deficit/hyperactivity disorder telemental health treatment study. Child Adolesc Psychopharmacol 26(8):662-671 